\title{
Ueber die Deutung der Pulscurven beim Valsalva'schen und Müller'schen Versuch.
}

Von

\section{Philipp Knoll.}

\author{
Hierzu Tafel V.
}

Ein unter gleicher Ueberschrift in diesem Archive (Bd. LVI S. 389 ff.) erschienener Aufsatz von E u gen Hirs ehmann veranlasst mich, darauf aufmerksam zu machen, dass ich schon im Jahre 1880 unter eingehender Erörterung der einschlägigen Verhältnisse Pulscurven vom Menschen bei beiden Versuchsarten veröffentlichte, welche im Wesentlichen mit den von $\mathrm{Hirschmann}$ unter Benutzung einer besonderen Versuchsanordnung vom morphinisirten Hunde gewonnenen ïbereinstimmen, und dass ich ein Jahr später die beim Vals alva'schen Versuche in Betracht kommenden Erscheinungen, einschliesslich der eintretenden Veränderung. der Pulsfrequenz, auch einer genaueren experimentellen Untersuchung unterzog.

Beide Arbeiten wurden aus örtlichen Rücksichten in einer, in medizinischen Kreisen wohl kaum bekannten Zeischrift (Lotos, Jahrbuch für Naturwissenschaft. Nene Folge. Bd. I u. II. Prag, bei Tempsky) veröffentlicht, was es wohl bedingt haben mag, dass dieselben, trotz der Berïcksichtigung in dem von $\mathrm{H}$ o f $\mathrm{m} \mathrm{a} \mathrm{n} \mathrm{n}$ und $\mathrm{S} c \mathrm{~h}$ w a 1 be herausgegebenen Jahresberichte, Autoren, welche sich später mit diesem Gegenstand beschäftigt baben, so v. Frey, Tra u t w e in und H i r s chmann, entgangen sind.

Mit Rïcksicht hierauf aber dürfte ein Wiederabdruck der Pulscurven, die ich mittels des von mir beschriebenen Polygraphen beim Valsalva'schen und M ii 11 e r'schen Versuch am Menschen seinerzeit aufgenommen, an dieser Stelle um so berechtigter erscheinen, als in denselben die Erscheinungsreihe, welche hierbei zu Tage treten kann, vollständiger zum Ausdruck kommt, als in irgend einer der anderen Publikationen, was einerseits in der Art der Ausführung der Versuche, anderseits in den benutaten technischen Mitteln begründet ist.

So tritt in Fig. 1, wo bei a, beim Valsalva'schen Ver- 
suche, auf der Höhe der Inspiration die active Exspiration bei Verschluss von Mund und Nase einsetzt, das durch die stossweise Ausführung dieser Exspirationsbewegung bedingte Auspressen von Blut in die extrathoracalen Arterien und die hierdurch hervorgerufene arterielle Drucksteigerung nicht bloss in einer Erhebung der ,Minima“ der Palseurven (Hirschmann), sondern anch in einer entsprechenden Formveränderung derselben hervor, während sich gleichzeitig anch schon die durch die Herzeompression bedingte, bis zum Schluss des Versuches anhaltende Verminderung des Pulsvolumens deutlich ausprägt.

Auch die rasch hierauf folgende $V$ erm inderung, sowie die bei längerer Andauer des Versuches eintretende, durch Wachsen der Pulsfrequenz sowie dyspnoisehe und anämische Vasoconstrictoren-Reizung bedingte Erhöhung des arteriellen Druckes spricht sich nicht blos in einem Sinken und Steigen der ,Minima“, sondern durehwegs auch in den entsprechenden Veränderungen der Form der Pulseurven aus.

Ebenso kommt bei der stossweisen Beendigung des Versuches die durch die plötzliche Entlastung der intrathoracalen Gefässe und die Aspiration von Blut aus den extrathoracalen Arterien bedingte fliichtige Erniedrigung des Druckes in letzteren sowie sein darauf folgendes, auf anbaltender Gefässverengerung beruhendes, später allmählich wieder den ursprünglichen Verhältnissen weichendes Wiederansteigen sowohl an der Lage der "Minima", als an den Pulscurvenformen zu deutlicher Ausprägung.

Auch die während des Versuches eintretende Beschlennigung des Herzschlages, welche auf einer durch Erregung sensibler Herznerven herbeigeführten Verminderung des Vagustonus beruht, sowie das nachher vorübergehend auftretende Seltenerwerden des Pulses, das wohl hauptsächlich auf eine postanämische Reizung des Vaguscentrums zu beziehen ist (Lotos, Bd. II. S. 9-20), sind wohl ausgeprägt.

Es braucht aber wohl kaum besonders darauf hingewiesen zu werden, dass es davon, ob der Versuch brüsk begonnen und beendet wird, ferner von der Dauer desselben und wohl auch von der Erregbarkeit der nervösen Centren des Versuchsindividuums abbängt, $o b$ alle geschilderten Erseheinungen und $o b$ sie in gleicher Dentlichkeit zn Tage treten wie hier. Es muss ferner betont werden, dass die bei diesem Versuche leicht eintretenden Mitbewegungen in den Muskeln der Extremität, an welehe der Sphygmo. 
graph angelegt ist, sorgfältig vermieden werden müssen, um das hier verzeichnete Ergebniss zu erlangen, was durch Uebung und Aufmerksamkeit beim Mensehen wohl gelingt, beim morphinisirten Versuchsthiere aber kaum mit Sicherheit zu erreichen sein dürfte.

Endlich ist die Art, wie der. Sphygmograph an der betreffenden Arterie angelegt ist, von wesentlicher Bedeutung für das bei diesem Versuche zu erlangende Sphygmogramm.

Hi rschmann, der, nach dem Vorgange v. Frey's, auf die Interferenz venöser Stauung und deren Einfluss auf das Volumen der Extremität verweist, führt aus, dass diese bei den gebräuchlichen Arten den Sphygmographen zu appliciren, zu einer „Elevation der Minima" führen mïsse.

Bei Verwendung des von mir beschriebenen Polygraphen ist dies aber nicht der Fall, da hier der Sphygmograph mittels einer Doppelschraubenklemme derart fixirt wird, dass der Arm an der Beugeseite nur dicht neben der Arterie, und an der Streckseite an analogen Stellen comprimirt wird, während die übrige Peripherie desselben frei bleibt und bei der etwa durch venöse Staung bedingten Schwellung leichter nachgiebt als die Gegend, wo die Arterie beziehungsweise der Sphygmograph liegt.

Man kann denn anch bei dieser Art der Application des Sphygmographen an der Cubitalis eine bis zu ausgeprägter Cyanose der Hand führende Umschnürung des Oberarmes vornehmen, ohne jede, oder nur mit einer kaum merkbaren „Elevation der Minima“.

An der bei Ausführung des Müller'schen Versuches am Menschen gewonnenen Fig. 2 prägen sich den vorher beschriebenen in vielen Punkten entgegengèsetzte Veränderungen, wenn auch in schwächerem Maasse aus. Dort wo der Versuch nach einer tiefen Ausathmung, die zu einer flüchtigen „Elevation der Minima“ der Pulscurven fïhrt mit einer starken, jähen Erweiterung des Brustraumes bei Verschluss von Mund und Nase einsetzt, bei a, findet man ein mässiges Absinken der „Minima“, das auf Aspiration von Blut aus den extrathoracalen Arterien zu beziehen ist. Bald darauf aber, entsprechend der vermehrten Zufuhr von Blut zum rechten und von da zum linken Herzen, sowie der, allerdings bei den einzelnen Individuen sehr verschieden ausgeprägten Zunahme der Pulsfrequenz, die wohl auf dem bekannten, durch Hering entdeckten Reflex von den sensiblen Lungennerven auf den Herzschlag beruhen dürfte, siebt man die "Minima" allmählich bis 
uber das frühere Niveau sich erheben und das Pulsvolumen etwas anwachsen, während in Uebereinstimmung hiermit, aber allerdings auch nur in ganz mässigem Grade, eine Abschwächung der ,Ruickstosselevation" und eine Verstärkung.der ,ersten Elasticitätselevation“ erkennbar wird.

\section{Erklärung der Figuren anf Tafel V.}

Die Pulscurven wurden mittels eines Transmissionssphygmographen, die darunter stehenden Athemcurven mittels eines in der Oberbauchgegend applicirten elastischen Sackes verzeichnet.

Die aufsteigende Linse zeigt an letzteren im Allgemeinen die Inspiration, die absteigende die Exspiration an. Doch prägt sich die kräftige Contraction der Bauchmuskeln während des $\mathrm{V}$ alsalva'schen Versuches, entsprechend der Vorwölbung der Oberbanchgegend, durch einen Hochstand, das Einsinken dieser Gegend bei der starken Erweiterung des unteren Brustraumes während des Müller'schen Versuches dagegen durch Tiefstand des Schreibhebels aus.

Nach Beendigung des letzteren Versuches tritt znerst eine starke Hervorwölbung der Oberbanchgegend und dann erst eine Ausathmung auf.

Die einzelnen Abschnitte der Abscisse haben den Zeitwerth von Secunden.

(Aus dem physiologischen Institut in Rostock.)

\section{Zur Lehre von der Rhythmicität des Herzmuskels.}

Nach gemeinschaftlich mit Herrn cand. med. D o b b e r t in angestellten Versuchen

von

\section{Langendorff.}

(Mit 1 Abbildung.)

„Es wankt - und fällt, wie es scheint - die alte Lehre, welche in dem eigenen Nervensystem des Herzens die Quelle der selbständigen Herzthätigkeit und den Vermittler des Herzrhythmus 